\title{
Evaluation of Undescended Testes in Newborns: It Is Really Simple, Just Not Easy
}

\author{
Martin Promm ${ }^{\mathrm{a}}$ Annika Dittrich ${ }^{\mathrm{a}}$ Susanne Brandstetter ${ }^{\mathrm{b}}$ \\ Sara Fill-Malfertheiner ${ }^{c}$ Michael Melter ${ }^{b}$ Birgit Seelbach-Göbel ${ }^{c}$ \\ Christian Apfelbacher ${ }^{d, e}$ Michael Kabesch ${ }^{b}$ Wolfgang H. Rösch ${ }^{a}$ \\ the KUNO-Kids Study Group
}

\begin{abstract}
aDepartment of Pediatric Urology, Clinic St. Hedwig, University Medical Center of Regensburg, Regensburg, Germany; bUniversity Children's Hospital Regensburg (KUNO-Clinics), University of Regensburg, Clinic St. Hedwig, Regensburg, Germany; ${ }^{~ C}$ Clinic of Obstetrics and Gynecology St. Hedwig, University of Regensburg, Regensburg,

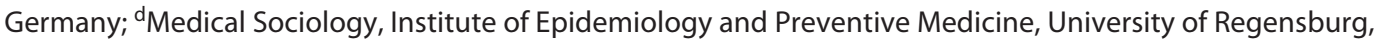
Regensburg, Germany; eInstitute of Social Medicine and Health Economics, Otto von Guericke University Magdeburg, Magdeburg, Germany
\end{abstract}

\section{Keywords}

Examination - Cryptorchidism · Hormone therapy ·

Orchidopexy $\cdot$ Retractile testis $\cdot$ Screening

\begin{abstract}
Introduction: The evaluation of the testicular position in newborns is important to ensure timely initiation of therapy. The aim of our study was to assess the reliability of a routinely performed screening examination. Patients and Methods: Newborns were examined by a pediatrician between 48 and $72 \mathrm{~h}$ after birth. Boys with suspected cryptorchidism were double-checked by a pediatric urologist within 24 h. Results: 1,181/2,353 children included in the study between June 2015 and December 2017 were male. Eight hundred sixty-one boys could be included in this analysis; $5.8 \%$ ( $n=50$ ) were diagnosed with undescended testis (UDT) by the pediatrician. 30/50 boys were double-checked at the Department of Pediatric Urology. Forty percent (20/50) were lost to follow-up. In 43\% (13/30), the diagnosis could be con-
\end{abstract}

karger@karger.com www.karger.com/uin

Karger $\stackrel{\text { ' }}{5}$

GOPEN ACCESS
(C) 2021 The Author(s)

Published by S. Karger AG, Basel

This is an Open Access article licensed under the Creative Commons Attribution-NonCommercial-4.0 International License (CC BY-NC) (http://www.karger.com/Services/OpenAccessLicense), applicable to the online version of the article only. Usage and distribution for commercial purposes requires written permission. firmed. Three former studies had shown a relevant discrepancy in the results of the diagnosis of UDT made by health care providers and urologists/pediatric surgeons. To our knowledge, this is the first study evaluating the testicular position in male newborns in such a large prospective birth cohort study by physicians with ranging expertise within 1 day. Conclusion: Further treatment for UDT is based on clinical examination. Ours and previous studies can clearly show the various findings in boys suspected having UDT. Therefore, it is essential that the diagnosis is confirmed by a specialist before a therapy is initiated.

(c) 2021 The Author(s)

Published by S. Karger AG, Basel

\section{Introduction}

With a prevalence of $2-4.6 \%$ in Western countries "undescended testis" (UDT) is one of the most common malformations in boys [1]. The evaluation of the testicular position is an essential part of the so-called baby check 
2353 KUNO kids study participations (boys $=1181$ )

Male participation with available data from screening $(n=861)$

Boys screened with undescended testes (UDT)

$(n=50)$

Boys presented to pediatric urologist $(n=30)$
Boys screened with descended testes $(n=811)$

Lost to follow-up $(n=20)$

Diagnose with UDT

was confirmed $(n=13)$

Diagnose with UDT

was not confirmed $(n=17)$

Fig. 1. Flowchart of patients.

(prevention screening program) of male infants in Germany performed by pediatricians or general practitioners (GPs). The evaluation of the testicular status is obligatory to ensure timely initiation of therapy to prevent consequential damage to the testes, especially infertility and development of testicular cancer. The extents to which the results of the examinations performed by pediatricians or GPs can be confirmed by a specialist is unknown. For the first time in Germany, we investigated how the results of the standard German neonatal screening performed by pediatricians and an examination by pediatric urologists match. In this prospective birth cohort study, boys screened postnatally for UDT were reevaluated by a pediatric urologist. The monocentric study was performed in a tertiary perinatal center that is part of the University of Regensburg with $>3,000$ births per year.

\section{Patients and Methods}

Data for this analysis were collected as part of a prospective multipurpose birth cohort study called KUNO-Kids. The aim of the study is to investigate a wide spectrum of exposures and outcomes [2]. The mother-child pair was included in the KUNOKids study immediately after birth. All participating parents provided written informed consent. Boys without complete questionnaires and written informed consent for the study itself as well as consent for physical examinations were excluded from our analysis. The newborns were assessed between 48 and $72 \mathrm{~h}$ after birth by a pediatrician according to a predefined standardized clinical procedure. This examination is part of an extended German prevention screening program and includes the evaluation of the testicular position and the documentation of birth weight and week of gestation. Boys screened with UDT in this examination were double-checked by a consultant of the Department of Pediatric Urology within $24 \mathrm{~h}$. Both examinations evaluating the testicular

Evaluation of Undescended Testes in Newborns position were made under standardized conditions in the supine position. The evaluations were performed by 5 different pediatricians and 2 different pediatric urologists. Data were assessed using paper-based and electronic case report forms. Descriptive statistics were calculated.

\section{Results}

Of 2,353 children included in the study between June 2015 and December 2017, 1,181 were male. For 861 boys data from the prevention screening program were available, and those boys could be included in this study. In the standard U2 screening 5.8\% $(n=50)$ were diagnosed with UDT. Thirty of these 50 boys screened with UDT were double-checked by a pediatric urological consultant within $24 \mathrm{~h}$ to verify the diagnosis. $20 / 50$ (40\%) of the male newborns screened with UDT were lost to follow-up due to discharge from the hospital before the pediatric urological examination was performed. In $13 / 30(43 \%)$, the diagnosis of UDT could be confirmed (Fig. 1). 3/13 (23\%) of the boys with UDT were born preterm and 10/13 (77\%) were full-term neonates. In $2 / 13$ (15\%), the birth weight was below $2,500 \mathrm{~g}$ and in $11 / 13(85 \%)$ higher than $2,500 \mathrm{~g}$. In $9 / 13$, the exact position of the testes in the previous clinical examination was not documented; in $2 / 13$ cases, the exact testicular position was evaluated differently; and in $2 / 13$, the testicular position was evaluated identically. In 17 of the 30 boys (57\%), the initial diagnosis of UDT was not confirmed: Table 1 shows the results of the 2 groups with regard to the testicular position for the 17 boys diagnosed with UDT by pediatricians and not verified by pediatric urologists. 
Table 1. Comparison of the testicular position in not verified UDT

\begin{tabular}{lll}
\hline$N(17)$ & Diagnosis pediatrician & Diagnosis ped urologist \\
\hline 7 & Cryptorchidism, not specified & Scrotal position both sides \\
4 & Not palpable testes both sides & Scrotal position both sides \\
2 & Cryptorchidism, not specified & Retractile testes both sides \\
2 & Inguinal position both testes & Scrotal position both sides \\
1 & Cryptorchidism, not specified & Retractile testis right \\
1 & Not palpable testis left & Scrotal position both sides \\
\hline & UDT, undescended testis. & \\
\hline
\end{tabular}

\section{Discussion}

The evaluation of the testicular position in newborns is performed routinely by physicians, in Germany, mostly pediatricians and GPs. Although the examination of the testes ostensibly seems to be simple, everyone who deals with this topic knows how difficult it can be to make a reliable diagnosis. In our study, in only $43 \%$ of boys screened positive for UDT by the prevention screening program, the diagnosis was confirmed by the pediatric urologists.

Previous studies also have shown that diagnosis of UDT differs immensely between different physicians. Already in 1989, Olsen [3] described an interobserver variation in the assessment of UDT. Thirty-seven boys aged 2-11 years referred to an outpatient clinic in Copenhagen, Denmark, were investigated in a prospective study. Position and motility of the testes in those 37 boys were checked on the same day in the supine and squatting position independently by the same 2 physicians, a consultant in urology and surgery and a senior registrar with several years of experience. Only in $13.5 \%$ of the patients, a complete agreement on all observations was reached.

Snodgrass et al. [4] evaluated the referral patterns of boys diagnosed with UDT in 2011. In this prospective observational study, 118 boys aged $0-10$ years, diagnosed with UDT, had been referred to a pediatric urologist in Dallas, USA, by primary health care providers. A standardized history assessment, visual inspection of the scrotum for symmetry, a physical examination, and a review of previously obtained imaging findings were documented. Forty-three percent of these boys had descended testes, $51 \%$ had UDT, and $6 \%$ had indeterminate findings at the time of reevaluation. In a subgroup of the boys aged $0-12$ months $(n=35)$, the diagnosis could be confirmed in $77 \%$, and $60 \%$ of them were diagnosed with UDT at birth.
In 2017, Mau et al. [5] reported about the problem to evaluate testicular status in children in Ottawa, Canada. Eight hundred ninety-four boys diagnosed with UDT were referred to the Department of Urology by family physicians. Fifty-one percent of them had physiological positioned or retractile testis and $26 \%$ had a palpable UDT.

Although these studies differ in timeframe, observer, and settings, they all can clearly show a relevant discrepancy in the results of the diagnosis of UDT. Our results confirm these findings, where in the majority of the boys referred to the Department of Pediatric Urology, the diagnosis of cryptorchidism could not be verified. In order to avoid mistakes due to individual preferences or changing circumstances, the examinations were performed under standardized conditions in the supine position. Furthermore, the examinations were performed within $24 \mathrm{~h}$ to exclude the differences in the examination findings may be due to ascension of the testes. To the best of our knowledge, this is the first study evaluating the testicular position in male newborns in such a large prospective birth cohort study by physicians of 2 different specializations within $24 \mathrm{~h}$. Our study is limited by a high rate of patients lost to follow-up. Also, the comparison of findings obtained from a screening setup and a regular examination may be afflicted with errors due to different diagnostic focuses. Another possible pitfall could be that the screening was performed by consultants less experienced with this entity than the physicians who performed the control examination.

Besides more specific clinical training in evaluation of UDT (e.g., supported by training videos), further prospective interdisciplinary studies are needed to develop appropriate standardized examination methods to ensure that all trained physicians dealing with this important clinical issue are able to admit these boys for adequate therapy according to guidelines and to avoid inadequate treatment. 


\section{Conclusion}

Further treatment for cryptorchidism, whether surgical or conservative, is based on the results of clinical examinations. The results of ours and previous studies can clearly show the various findings in boys suspected having UDT by differently experienced physicians. Therefore, in our opinion, it is essential that the diagnosis of UDT must be confirmed in an appropriate standardized examination by an experienced specialist before a therapy is initiated.

\section{Acknowledgment}

The authors would like to thank all families who participated in the KUNO-Kids birth cohort study as well as all medical students, nurses, midwives, physicians, and researchers who facilitated the recruitment of participants and data assessment. Further, they thank all members of the KUNO-Kids study group: Petra Arndt (ZNL Transfer Center of Neuroscience and Learning, University of Ulm, Ulm, Germany), Andrea Baessler (Department of Internal Medicine II, Regensburg University Medical Center, Regensburg, Germany), Mark Berneburg (Department of Dermatology, University Medical Centre Regensburg, Regensburg, Germany), Stephan Böse-O'Reilly (Institute and Clinic for Occupational, Social and Environmental Medicine, University Hospital, LMU Munich, Munich, Germany), Romuald Brunner (Clinic of Child and Adolescent Psychiatry, Psychosomatics and Psychotherapy, Bezirksklinikum Regensburg [medbo], Regensburg, Germany), Wolfgang Buchalla (Department of Conservative Dentistry and Periodontology, University Hospital Regensburg, University of Regensburg, Regensburg, Germany), Sara FillMalfertheiner (Clinic of Obstetrics and Gynecology St. Hedwig, University of Regensburg, Regensburg, Germany), André Franke (Institute of Clinical Molecular Biology, Christian-AlbrechtsUniversity of Kiel, Kiel, Germany), Sebastian Häusler (Clinic of Obstetrics and Gynecology St. Hedwig, University of Regensburg, Regensburg, Germany), Iris Heid (Department of Genetic Epidemiology, University of Regensburg, Regensburg, Germany), Caroline Herr (Bavarian Health and Food Safety Authority [LGL], Munich, Germany), Wolfgang Högler (Department of Pediatrics and Adolescent Medicine, Johannes Kepler University Linz, Linz, Austria), Sebastian Kerzel (Department of Pediatric Pneumology and Allergy, University Children's Hospital Regensburg [KUNO], St. Hedwig Campus, Regensburg, Germany), Michael Koller (Center for Clinical Studies, University Hospital Regensburg, Regensburg, Germany), Michael Leitzmann (Department of Epidemiology and Preventive Medicine, University of Regensburg, Regensburg, Germany), David Rothfuß (City of Regensburg, Coordinating Center for Early Interventions, Regensburg, Germany), Wolfgang Rösch (Department of Pediatric Urology, University Medical Center, Regensburg, Germany), Bianca Schaub (Pediatric Allergology, Dept. of Pediatrics, Dr. von Hauner Children's Hospital, University Hospital, LMU Munich, Munich, Germany), Bernhard H.F. Weber (Institute of Human Genetics, University of Regensburg, Regensburg, Germany), Stephan Weidinger (De- partment of Dermatology, Venereology and Allergy, University Hospital Schleswig-Holstein, Campus Kiel, Kiel, Germany), and Sven Wellmann (Department of Neonatology, University Children's Hospital Regensburg [KUNO], St. Hedwig Campus, Regensburg, Germany).

\section{Statement of Ethics}

The KUNO-Kids birth cohort study was approved by the Ethics Committee of the University of Regensburg (File No. 14-1010347). Written informed consent was obtained for all participants from all legal guardians, and consenting to physical examinations of the child was necessary to be included in our analysis. We confirm that all research was performed in accordance with relevant guidelines and regulations in evaluating testicular position.

\section{Conflict of Interest Statement}

The authors declare that they have no conflicts of interest.

\section{Funding Sources}

The KUNO-Kids study was funded by research grants of the EU (HEALS: 603946) and the German Federal Ministry for Education and Research (SYSINFLAME: 01ZX1306E). Further financial support was provided by the University Children's Hospital of the University of Regensburg (KUNO-Clinics) and the clinic "St. Hedwig" (Hospital "Barmherzige Brüder Regensburg"). The funding bodies had neither influence on the design of the study, on the collection, analysis, and interpretation of data, nor in the writing of the manuscript.

\section{Author Contributions}

M.P. contributed to data analysis, examination of participants, drafting and evaluation of the questionnaire, manuscript writing, and revision and submission of the manuscript. He critically evaluated the manuscript and approved the final manuscript as submitted. A.D. recruited participants for the KUNOKids birth control study. She worked on drafting and evaluation of the questionnaires, as well as transmitting the paper-based questionnaires to an IT-based network. Furthermore, she contributed to data analysis and presented the results on a national pediatric congress. She critically evaluated the manuscript. S.B. designed the study, performed data analysis, interpreted the study findings, drafted the manuscript, critically evaluated the manuscript, and approved the final manuscript as submitted. S.F.-M. contributed to data collection, critically evaluated the manuscript, and approved the final manuscript as submitted. B.S.-G. contributed to data collection, critically evaluated the manuscript, and approved the final manuscript as submitted. M.M. contributed to data collection, critically evaluated the manuscript, and approved the final manuscript as submitted. M.K. 
contributed to data collection, data interpretation, and drafting of the manuscript. He critically evaluated the manuscript and approved the final manuscript as submitted. C.A. designed the study. He contributed to data collection, critically evaluated the manuscript, and approved the final manuscript as submitted.
W.H.R. designed the study and contributed to data analysis. $\mathrm{He}$ interpreted the study findings, drafted the manuscript, critically evaluated the manuscript, and approved the final manuscript as submitted.

\section{References}

1 Thorup J, McLachlan R, Cortes D, Nation TR, Balic A, Southwell BR, et al. What is new in cryptorchidism and hypospadias-a critical review on the testicular dysgenesis hypothesis. J Pediatr Surg. 2010;45(10):2074-86.

2 Brandstetter S, Toncheva AA, Niggel J, Wolff C, Gran S, Seelbach-Göbel B, et al. KUNOKids birth cohort study: rationale, design, and cohort description. Mol Cell Pediatr. 2019; 6(1):1-10.
3 Olsen LH. Inter-observer variation in assessment of undescended testis. Analysis of kappa statistics as a coefficient of reliability. $\mathrm{Br} \mathrm{J}$ Urol. 1989;64(6):644-8.

4 Snodgrass W, Bush N, Holzer M, Zhang S. Current referral patterns and means to improve accuracy in diagnosis of undescended testis. Pediatrics. 2011;127(2):e382-8.

5 Mau EE, Leonard MP. Practical approach to evaluating testicular status in infants and children. Can Fam Physician. 2017;63:432-5. 